\title{
Social Anxiety and Self-Compassion in Persons with Alcohol Use Disorders in Ghana
}

\author{
Marie Pearl Agordzo $^{1 *}$, Joseph Kwarteng Ofosuhene-Mensah ${ }^{2}$, Kofi Krafona ${ }^{3}$ and George Ekem-Ferguson ${ }^{4}$ \\ ${ }^{1,2,3}$ Department of Education and Psychology, University of Cape Coast, Ghana \\ ${ }^{4}$ Korle-bu Teaching Hospital, Accra-Ghana \\ Corresponding Author*
}

\begin{abstract}
Alcohol use disorder is a condition that develops as a result of problematic alcohol use. The study examined the relationship between social anxiety and self-compassion among persons with alcohol use disorder in the three main psychiatric hospitals and an alcohol rehabilitation centre in Ghana. The study employed a correlational design and used purposive sampling in obtaining its participants. Sixty participants were involved in the study and Frequencies and Pearson's productmoment correlation coefficient were used to analyse the data. The findings revealed that social anxiety was highly prevalent among participants and moderate level of self-compassion was observed. A highly significant inverse relationship between social anxiety and self-compassion was also found. The sample size and the use of an adapted version of the self-compassion scale are considered limitations for the study. Also, persons with alcohol use disorders in the study were addicted to at least one other psychoactive substance and this was also considered a limitation of the study.
\end{abstract}

Keywords: Alcohol use disorder, social anxiety, self-compassion.

\section{INTRODUCTION}

A lcohol is a psychoactive substance that has benefits which cannot be overemphasized. Its use cuts across gender, culture and race with its medicinal value reported (Stolberg, 2006). Even though the benefits of alcohol have been acknowledged (Dunbar, Launay, Wlodarski, Robertson, Pearce, Carney et. al., 2016), its harmful effects have also been documented. The harmful effects of alcohol have been implicated in every 1 out 20 deaths in 2016 and more than 5\% of the global disease burden (World Health Organisation (WHO), 2018). In Ghana, it has been estimated that about 1.2 million people suffer from alcohol and other drug related problems (Ofori-Atta, Read \& Lund, 2010).

Alcohol use disorder has been defined by Kranzler and Soyka (2018) as a problematic pattern of compulsive and uncontrolled alcohol use associated with clinically significant impairment or distress as defined by the Diagnostic and Statistical Manual for Mental Disorders V (DSM V). The DSM V spells out eleven criteria and requires that a person meets at least two out of these eleven criteria to merit the diagnosis of alcohol use disorder. However, the severity of the condition depends on number of criteria the person meets (APA, 2013).

Alcohol has been found to cause neuroadaptive changes to the brain upon continual usage (Koob \& Le Moal, 2008). This explains alcohol as a psychoactive substance that could serve both physiological and psychological purposes for its users (Dunbar, Launay, Wlodarski, Robertson, Pearce, Carney et. al., 2016). One of these purposes is the anxiolytic effect of the substance. This property of alcohol provides an avenue for people who experience physiological or psychological symptoms of anxiety to experience a feeling of relaxation especially in situations that trigger anxiety.

Social anxiety is a type of anxiety disorder described by the Diagnostic and Statistical Manual for Mental Disorders V to be marked with fear or anxiety about one or more social situations in which the individual is exposed to possible scrutiny by others (APA, 2013). The individual fears he or she may act in a way or show anxiety symptoms that will be embarrassing and humiliating. Social anxiety has been found to have comorbidity with alcohol use disorder and as such common among people with alcohol use disorder (Clarke \& Sayette, 1993: Kushner, Abrams \& Borchardt, 2000: Randall, 2000: Schellekens, De Jong, Buitelaar \& Verkes, 2014). The World Health Organisation (2019) report on harmful use of alcohol indicated that persons with alcohol use disorders who have comorbid anxiety disorders are likely to relapse during the first three months of treatment.

In Africa and specifically Ghana alcohol was used as an expression of masculinity and was consumed by the affluent, people who had status and were of a high social class in society (Akyeampong, 1995). In recent times, this trend has changed significantly. The marketing of alcoholic beverages has seen a spike in media advertisement. This is due to the absence of an implemented national policy on alcohol (WHO, 2011). Commercials in the media portray alcohol as a need with celebrities and high profile personalities playing major roles. Currently, there are variety of these alcoholic beverages on the Ghanaian market and each portrays its potency without disclosing the long term effects. These drinks are also relatively more affordable than most soft drinks on the market (Barry, Johnson, Rabre, Darville, Donova \& Efunbumi, 2015). These, coupled with the upsurge of numerous bars and spots has seen the easy availability of the substance and lot of patronage by even the unemployed youth.

Similar to most psychological conditions, alcohol use disorder is not a mere national problem but a global issue (Fisher, Bang \& Kapiga, 2007). However, in Ghana, alcohol use 
disorder is often viewed as a moral problem and people in this situation are often judged based on social values and are perceived to have weak morals. The moral definition of the condition often makes society critical of such persons with labels such as 'korinsani, dantorlor' (literally meaning drunkard) among others used to describe them. However, the underlying cause of the condition includes mental health related issues such as anxiety and depression (Appiah, Danquah, Nyarko, Ofori-Atta \& Aziato, 2017: OppongAsante \& Kugbey, 2019).

In the face of the challenges posed by definition of alcohol use disorder in Ghana and the critical attitude towards alcohol use disorder within the Ghanaian context, there is a need for people with alcohol use disorder to find strategies to cope with their situation. This is particularly because their condition is one that is often looked upon generally with disdain. One of these strategies is to develop self-compassion. Neff (2004) opined that self-compassion is an emotional regulation strategy where people accept themselves in an understanding, kind and humane way. This enables people suffering various forms of psychological distress to view their negative state in a positive light.

Neff (2009a) reported a link between self-compassion and psychological health including happiness, conscientiousness, optimism, decreased anxiety, depressive symptomology and rumination. According to Neff self-compassion is the ability to treat oneself with kindness, recognising one's shared humanity and being mindful when considering one's negative aspects. This eventually acts as a buffer against anxiety (Neff, Kirkpatrick \& Rude, 2007). Neff (2003a: 2003b) further indicated being self-compassionate makes it less likely to use alcohol to cope with anxiety.

Even though a few studies such as Akyeampong (1995) found social anxiety to exist among persons with alcohol use disorders in Ghana, there is the need to investigate the prevalence of social anxiety among persons undergoing treatment for alcohol use disorder. This is necessary because relapse is common among such people (Appiah , Boakye, Ndaa \& Aziato, 2017) and social anxiety has been implicated as a cause (World Health Organisation, 2019). There is also the need to find out the prevalence of self-compassion among these persons. This has become necessary as these people would be integrated into communities after treatment with less expectation of relapse and self-compassion could be beneficial for such purpose (Neff, 2003a: 2003b). Knowledge from the findings from this study would be beneficial to both researchers and practitioners in the field of substance disorders. Also, findings from the study would help the Mental Health Authority and Drug Rehabilitation Homes include appropriate and effective psychosocial skills into treatment regimens to avoid relapse.

\section{METHODS}

\section{Design and setting}

The study adopted a correlational design. This allowed for the relationship between social anxiety and self-compassion to be established among a clinical sample of persons with alcohol use disorder. Data for the study was collected from Ankaful Mental Hospital, Accra Psychiatric Hospital, Pantang Mental Hospital and House of Saint Francis, a rehabilitation centre where patients were receiving treatment.

\section{Participants}

This study received ethical clearance from the University of Cape Coast Institutional Review Board. There were sixty (60) participants obtained through purposive sampling for the study. Sixteen (16) participants were obtained from Ankaful Mental Hospital, six (6) from Accra Psychiatric Hospital, twenty-one (21) from House of Saint Francis Rehab and seventeen (17) from Pantang Mental Hospital. Each participant in the study had to meet the requirements of an inclusion criteria to be enrolled in the study. The criteria detailed that a participant of the study should be clinically diagnosed of alcohol use disorder from the clinical facility without drug induced psychosis and should be in treatment for at least a week.

\section{Measures}

To measure social anxiety, the Social Interaction Anxiety Scale (SIAS) by Mattick and Clarke (1998) was adopted. The instrument is a 20 item questionnaire on a 5 point Likert scale ranging from 'not at all' to 'extremely'. Mattick and Clark found a strong internal consistency of $a=0.84$ in both clinical and undergraduate samples. The Cronbach alpha for the scale in the sample for this study was $a=0.92$. The scale has three categorisations based on the scores obtained. The first category involved people with normal level of anxiety with a score of below 34, the second category involved persons who obtained a score ranging from 34 to 42 and they were categorised as social phobics while a score of 43 and above indicated social anxiety.

In measuring self-compassion, the Self-Compassion Scale developed by Neff (2003a) was adapted. The original instrument consists of 26 items on a 5-point Likert scale ranging from 'almost never' to 'almost always'. It has six facets that determine the presence or absence of selfcompassion. The facets are self-kindness, self-judgement, common humanity, isolation, mindfulness and over identification. The Self-Compassion scale used in this study was an adaption of the original 26 items to a 23 item. Cronbach alpha of the 23 item scale was found in the study to be 0.7 .

The items 'When times are really difficult I tend to be tough on myself', 'when I'm feeling down, I tend to feel like other people are probably happier than I am' and 'when I fail at something that is important to me, I tend to feel alone in my 
failure' were deleted. For the purpose of this work, the scale had three categorisations, scores that were one standard deviation below the mean were indicative of a low selfcompassion. Those that were one standard deviation above the mean were also indicative of a high self-compassion, any score that lied within the low or high self-compassion values were indicative of a moderate self-compassion.

Table 1: Sample characteristics $(\mathrm{N}=60)$

\begin{tabular}{|c|c|}
\hline Demographic & $N$ \\
\hline \multicolumn{2}{|l|}{ Sex } \\
\hline Male & 56 \\
\hline Female & 4 \\
\hline \multicolumn{2}{|l|}{ Age } \\
\hline Below 30 years & 23 \\
\hline $31-40$ years & 24 \\
\hline $41-50$ years & 7 \\
\hline 51 years and above & 6 \\
\hline $\begin{array}{l}\text { Marital Status } \\
\text { Single }\end{array}$ & 42 \\
\hline Married & 12 \\
\hline Divorced & 2 \\
\hline Separated & 4 \\
\hline \multicolumn{2}{|l|}{ Duration in Treatment } \\
\hline Below 4 weeks & 4 \\
\hline 5-6 weeks & 5 \\
\hline 7-8 weeks & 2 \\
\hline 9-10 weeks & 2 \\
\hline 11-12 weeks & 15 \\
\hline 13 weeks and above & 32 \\
\hline \multicolumn{2}{|l|}{ Religion } \\
\hline Christian & 55 \\
\hline Muslim & 4 \\
\hline Traditional & 1 \\
\hline
\end{tabular}

${ }^{\mathrm{a}} \mathrm{S}$ ample include 60 inpatients receiving treatment for alcohol use disorder

Table 2: Mean and Standard Deviations of Variables $n=60$

\begin{tabular}{|c|c|c|}
\hline & Mean & SD \\
\hline Social Anxiety & 43.77 & 11.39 \\
\hline Self-Compassion & 74.05 & 11.15 \\
\hline
\end{tabular}

Score of the participants on the social anxiety scale indicated that majority of them experienced high social anxiety and moderate self-compassion. The percentage distributions for the social anxiety and self-compassion are represented on figures I and II respectively. Figure I shows that $55 \%$ of the participants had social anxiety.

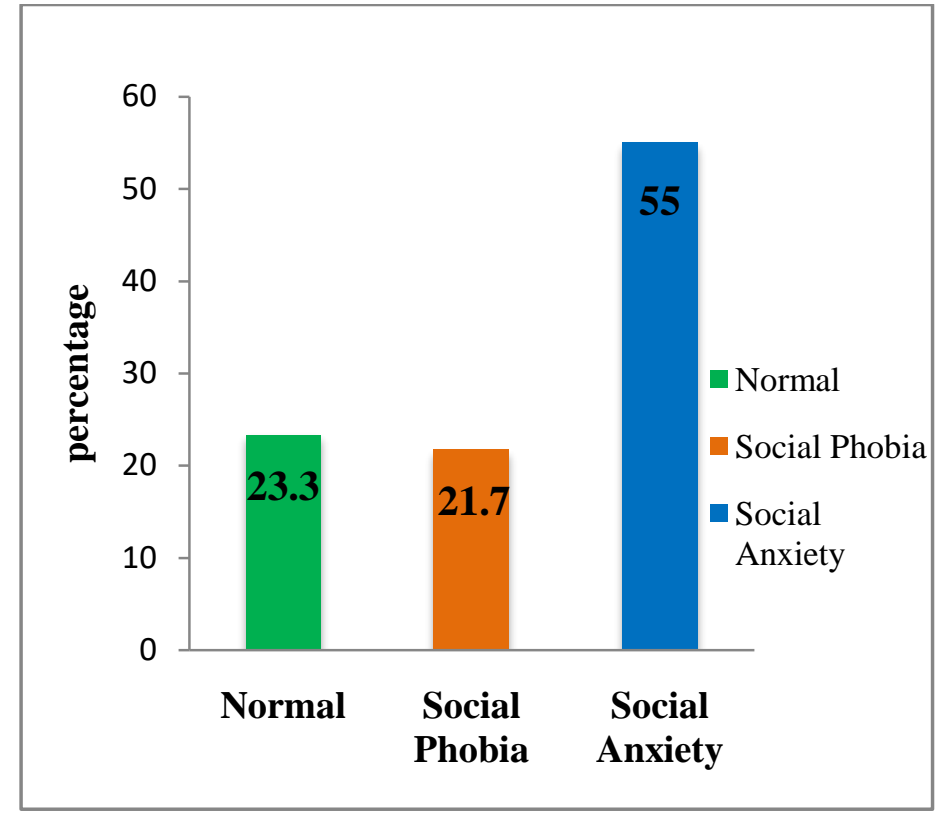

Figure I: Percentage Distribution of Social Anxiety

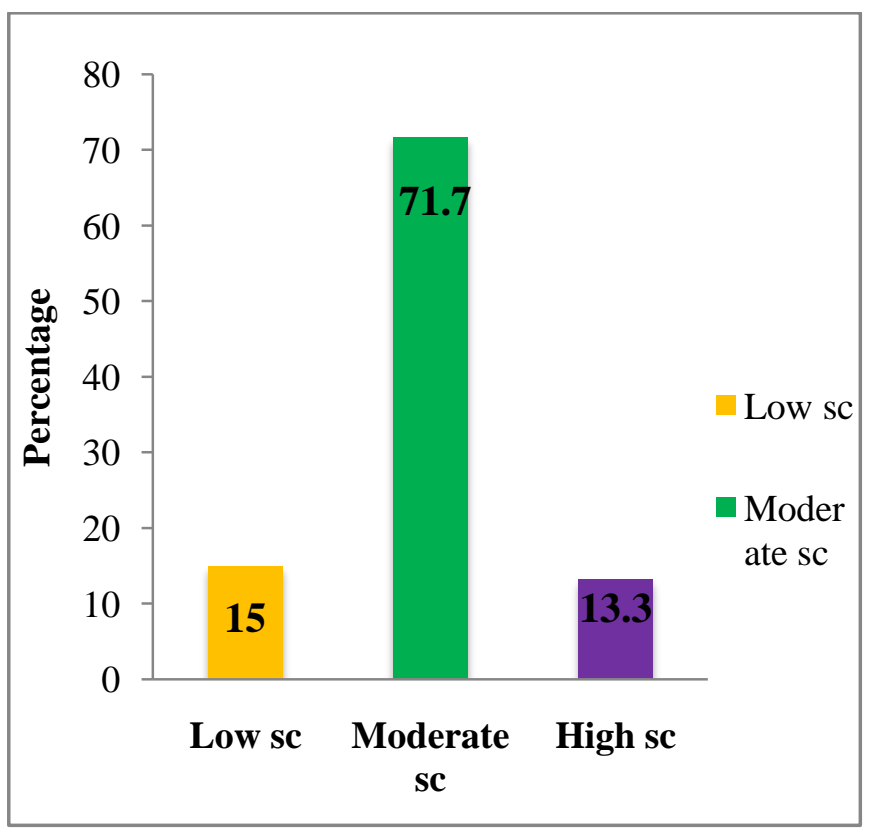

Figure II: Percentage Distribution of Self Compassion

The relationship between social anxiety (as measured by Social Interaction Anxiety Scale) and self-compassion (as measured by Self-Compassion Scale) was investigated using the Pearson product moment correlation co-efficient. Preliminary analyses were performed to ensure no violations of assumption of normality, linearity and homoscedasticity. There was a moderate significant negative relationship between social anxiety and self-compassion, $(r=-.44, n=60$, $p<0.01)$, with high levels of social anxiety associated lower levels of self-compassion. This implies that social anxiety is a significant factor that influences self-compassion. Interestingly, the correlation co-efficient $\left(\mathrm{r}^{2}\right)$ between social 
anxiety and self-compassion was 0.19 which implies that social anxiety helps to explains nearly $19 \%$ of the variance in participants score on self-compassion.

Table 3: Relationship between social anxiety and self-compassion

\begin{tabular}{|c|c|c|}
\hline & 1 & 2 \\
\hline 1. Social anxiety & & $-.44 * *$ \\
\hline Self-compassion & & \\
\hline
\end{tabular}

** correlation significant at 0.01

\section{DISCUSSION}

That majority of the participants were males and this finding is consistent with the assertion of APA (2013) and Akyeampong (1995) who argued that alcohol use disorder is a male dominated condition and was used express power especially for Ghanaian man respectively. Again, males are expected to play major roles in their various families, societies and communities compared to their female counterparts. Magazine (2004) found that men are more likely to drink heavily because drinking helps them to ignore responsibilities especially domestic roles while women have also been found to be less likely to drink because they are saddled with greater domestic responsibilities (Ahlstrom, Bloomfield \& Knibbe, 2001). Drinking may therefore hamper their efficiency in carrying out the enormous demands and responsibilities they must perform on a daily basis to run the home.

It must also be mentioned that the Ghanaian culture frowns on female alcohol use especially when a female develops an alcohol use disorder. Although women have been found to be alcohol users with some having alcohol related problems in Ghana (Agoabasa, 2012), cultural expectations which frown on women alcohol use, limits female drinking. Despite female occasional and low dose drinking being permissible; females are subjected to severer judgement when they develop alcohol use disorder. Furthermore, women also go through periods of pregnancy and child delivery at certain periods in their lives. These periods are known to be very crucial and exposures to alcohol have been found to have detrimental effects on the child (WHO, 2016). This could possibly explain the gender difference observed in the study.

It was found that younger persons were more implicated than the elderly. Over $78 \%$ of the respondents were less than 41 years. This finding was similar to that found by Adu-Mireku (2003) and Doku, Koivusilta and Rimpala (2012) as they called the condition a growing concern in Ghana and said concerted efforts was needed to curb the situation. This trend was an emerging problem observed by the World Health Organisation in 2011. They found that Ghanaian college students ranked third in Africa for problematic alcohol use on the continent. The WHO report was more disturbing as it implicated persons in college who are expected to know the devastating effects of alcohol and lead the campaign to educate Ghanaians on the addictive nature of the substance and the possible harm it causes in the long term.

It was again observed that most of the respondents in this study were single compared to the married, divorced and separated respondents. The distribution was a reflection of a finding by Schonbrun, Zach, Stuart \& Strong (2011). They asserted that marriage was a buffer against alcohol use disorder. Beig single is a state that could render one vulnerable to alcohol use as the support married people may obtain from their spouses in times of psychological distress would be absent for these individuals. This could account for the high frequency of single persons being implicated in the condition. In addition to this, since the condition is morally defined in the Ghanaian context, it is possible that people may not want to marry with individuals perceived to have weak morals.

The demographic data also provided information on participants' religion. A report from Ghana Statistical Service (2013) indicates that most Ghanaians are religious and indicate to state to be either Christians (71.2\%) or Moslems (17.6\%) or the African Traditional Religion (5.2\%). In a similar sense, most of the respondents in the study were Christians. This could be due to the greater proportion of the Ghanaian populace being Christians. In addition to this, Islamic religion compared to the Christian religion strongly forbids the use of alcohol. It is also possible that the sufferers of the condition in the Islamic religion may not want to disclose their state in order to preserve their identity and integrity as Moslems.

The study found that majority of the scores of participants indicated social anxiety. This is supported by studies on alcohol use disorder and social anxiety which indicate that alcohol use disorder and social anxiety are usually comorbid disorders (Kushner, Abrams \& Borchardt, 2000; Clarke \& Sayette, 1993). Akyeampong (1996) also reported in his study in Ghana that most people with social anxiety are highly likely to use alcohol especially for the relaxing effects that alcohol provides. It is also possible that persons with alcohol use disorders have high social anxiety as a reaction to the cultural outlook on the condition in Ghana.

It was also observed that majority of respondents scored within the moderate range for self-compassion. This is an indication of respondents' empathy and kindness they demonstrate towards themselves in spite of their condition. In Ghana, chronic medical and psychological conditions including alcohol use disorder have been given definitions such as the supernatural which placed the individual at the mercy of the 'gods' or a deity. In a study by Agoabasa (2012) it was found that some persons with alcohol use disorder in Ghana believed that a pot had been deposited in their belly which called for the continual use of the substance. A spiritual explanation as this makes it easy to empathise with oneself and treat oneself with more kindness and concern as the persons with alcohol use disorder perceive themselves as 
victims of the condition. In another study by Appiah, Boakye, Ndaa and Aziato (2017), they found spiritual explanations being offered to the cause of substance abuse. This further provides possible explanation to the moderate selfcompassion observed in the study.

A negative correlation between social anxiety and selfcompassion was observed in the study. A similar findig was made by Werner, Jazaieri, Goldin, Ziv, Heimberg and Gross (2012). They found that persons with social anxiety disorder reported significantly lower scores on self-compassion than healthy controls. In another study by Potter, Yar, Francis and Schuster (2014), it was found that self-compassion negatively correlated with social anxiety. Self-compassion has been found to involve how an individual thinks of himself considering his/her humanity and making allowances for error. Self-compassionate thoughts and behaviours are regarded to be positive and enhancing and they shield an individual from engaging in counterproductive thoughts as a result of being critical on oneself.

According to Leary, Tate, Adams, Allen and Hancook (2007) people diagnosed of suffering from anxiety are often cushioned against feelings of anxiety when they tend to be self-compassionate even though they may keep the anxiety provoking situations in focus. This result was found after their subjects recalled an event that involved failure and embarrassment. They found that after a treatment involving self-compassionate meditation, these persons reported less negative affect. In a recent study by Blackie and Kocovski (2017) they found that social anxiety as measured by social interaction anxiety scale and self-compassion as measured by self-compassion scale were negatively correlated. These studies suggest that self-compassion provided a buffer against the feeling of social anxiety disorder and was similarly reported in this study.

People experiencing social anxiety are often preoccupied with self-defeating thoughts which lies parallel to those in selfcompassion. Having mentioned this, it is worthy to note that even though the Ghanaian culture is lenient with alcohol use, it frowns on people with alcohol use disorder. Such persons are often considered as moral failures and social misfits. These individuals are likely to respond based on this cultural and societal judgement and tend to be critical of themselves, eventually avoid instances that puts them in the public space for fear of being negatively evaluated. This puts such persons at risk of developing social anxiety disorder and consequently uses alcohol or possibly relapses. In the absence of the skill of being self-compassionate, this phenomenon hampers the efforts of both the patients and the clinicians or counsellors as relapse becomes inevitable.

Based on the high prevalence of social anxiety observed from the results of the study and the effect of social anxiety on the success of treatment and relapse prevention, it is necessary that clinicians and counsellors attending to people with alcohol use disorder inculcate self-compassion meditation skills in the treatment regimen. This is to act as a cushion for these persons especially after treatment in order to limit the occurrence of relapse.

\section{CONCLUSIONS}

Alcohol use disorder is a condition that develops as a result of problematic alcohol use which has far reaching psychological consequences on the individual and society. Our study found a high prevalence of social anxiety to exist among persons with alcohol use disorders in Ghana, with moderate levels of self-compassion. The study further found a significant negative relationship between social anxiety and selfcompassion among persons with alcohol use in Ghana. Considering the high prevalence of social anxiety observed in the findings of this study, the study recommended that selfcompassion meditation exercises are included in the treatment of persons with alcohol use disorders. It is also recommended that further studies be conducted to find the influence of social anxiety on self-efficacy to resist alcohol among persons with alcohol use disorders in Ghana.

\section{REFERENCES}

[1] Adu-Mireku, S. (2003). Prevalence of alcohol, cigarette, and marijuana use among Ghanaian senior secondary students in an urban setting. Journal of Ethnicity in Substance Abuse, 2(1), 5365.

[2] Agoabasa, F.A. (2012). Alcoholism among women and its impact on the socio-economic development of Ghana: A case study of Sandema. Unpublished Bachelor of Science Dissertation. Ashesi University, Ghana.

[3] Ahlstrom, S., Bloomfield, K., \& Knibbe, R. (2001). Gender differences in drinking patterns in nine European countries: Descriptive findings.

[4] Akyeampong, E. (1996). What's in a drink? Class struggle, popular culture and the politics of akpeteshie (local gin) in Ghana, 1930-67. Journal of African History, 37(1), 215-236.

[5] Akyeampong, E. (1995). Alcoholism in Ghana: A socio- cultural exploration. Culture Medicine and Psychiatry, 19(2), 261-80. Substance Abuse, 22, 69-85.

[6] American Psychiatric Association (2013). Diagnostic and Statistical Manual of Mental Disorders (5 ${ }^{\text {th }}$ Ed) (DSM-5). Arlington, VA: Author.

[7] American Psychiatric Association (2004). Diagnostic and Statistical Manual of Mental Disorders, (4 ${ }^{\text {th }}$ ed) (DSM-4). Washington DC: Author.

[8] Appiah, R., Danquah, S., Nyarko, K. Ofori-Atta, A.L. \& Aziato L. (2017). Precipitants of substance abuse relapse in Ghana: A qualitative exploration. Journal of Drug Issues, 47(1), 104-115.

[9] Barry, A.E., Johnson, E., Rabre, A., Darville, G., Donovan, K.,M. \& Efunbumi, O. (2015). Underage access to to online alcohol marketing content: a Youtube case study. Alcohol and Alcoholism, 50(1), 89-94.

[10] Blackie, R.A., \& Kocovski, N.L (2017). Examining the relationships among self-compassion, social anxiety and postevent processing. Psychological Reports. O(0), 1-21.

[11] Clark, D. B., \& Sayette, M. A. (1993). Anxiety and the development of alcoholism: Clinical and scientific issues. American Journal on Addictions, 2, 59-76.

[12] Doku, D., Koivusilta, L., \& Rimpala, A. (2012). Socioeconmic differences in alcohol and drug use among Ghanaian adolescents. Addictive Behaviours, 37(3), 357-360.

[13] Dunbar, R.I.M., Launay, J., Wlodarski, R., Robertson, C., Pearce, E., Carney, J., \& MacCarron, P. (2016). Functional benefits of (Modest) Alcohol Consumption. Adaptive Human Behavior and Physiology, 3(2). 
[14] Fisher, J. C., Bang, H., \& Kapiga, S. H. (2007). The association between HIV infection and alcohol use: A systematic review and meta-analysis of African studies. Sexually Transmitted Diseases, $34,856-863$.

[15] Ghana Statistical Service (2013). National Analytical Report. In 2010 Population and Housing Census: Summary Report of Final Result. Accra, Ghana: Ghana Statistical Service.

[16] Koob, G.F., \& Le Moal, M. (2008). Neurobiological mechanisms for opponent motivational processes in addiction. Philos Trans $R$ Soc Lond B Biol Sci. 363, 3113-3123.

[17] Kranzler, H., \& Soyka, M. (2018). Diagnosis and pharmacotherapy of alcohol use disorder: A review. The Journal of American Medical Association. 320(8), 815.

[18] Kushner, M. G., Abrams, K., \& Borchardt, C. (2000). The relationship between anxiety disorders and alcohol use disorders: a review of major perspectives and findings. Clinical Psychology Review, 20 (2), 149-171.

[19] Leary, M.R., Tate, E.B., Adams, C.E., Allen, A.B., \& Hancook, J. (2007). Self-compassion and reactions to unpleasant events: Then implications of treating oneself kindly. Journal of Personality and Social Psychology. 92, 887-904.

[20] Magazine, R. (2004). Both Husbands and banda (gang) members: Conceptualizing marital conflict and instability among young rural migrants in Mexico City, Men and Masculinities, 7, 144-165.

[21] Neff, K.D. (2009a). The role of self-compassion in development: A healthier way to relate with oneself. Human Development, 52(4), 211-214.

[22] Neff, K.D. (2004). Self-compassion and psychological well-being. Construct Human Sci. 9, 27-37.

[23] Neff, K. D. (2003b). Self-compassion: An Alternative Conceptualization of a Healthy Attitude Toward Oneself. Self \& Identity, 2(2), 85-102.

[24] Neff, K. D. (2003a). The Development and Validation of a Scale to Measure Self-compassion. Self \& Identity, 2(3), 223-250.

[25] Neff, K.D, Kirkpatrick, K.L., \& Rude, S.S. (2007). Selfcompassion and adaptive psychological functioning. Journal of Research in Personality, 41(1), 139-154.
[26] Oppong Asante, K., \& Kugbey, N. (2019). Alcohol use by schoolgoing adolescents in Ghana: prevalence and correlates. Mental Health and Prevention. 13, 75-81.

[27] Potter, R.F., Yar, K., Francis, A.J.P., \& Schuster, S. (2014). Selfcompassionmediates the relationship between parental criticism and social anxiety. International Journal of Psychology and Psychological Therapy. 14, 33-43.

[28] Randall, C.L. (2000). Alcoholism and social anxiety disorder. Paper presented at the annual meeting of the Research Society on Alcoholism, Denver, CO.

[29] Schellekens, A.F.A., De Jong, C.A.J., Buitelaar, J.K., \& Verkes, R.J. (2014). Co-morbid anxiety disorders predict early relapse after inpatient alcohol treatment. Eur Psychiatry, 30(1), 128-36.

[30] Schonbrun, Y.C., Zach, W., Stuart, G.L. \& Strong, D.R. (2011). Marital status and treatment utilization for alcohol use disorders. Addictive Disorders and their Treatment, 10 (3).

[31] Stolberg V.B. (2006). A review of perspectives of alcohol and alcoholism in the history of American health and medicine. J Ethn Subst Abuse, 5 (4), 39-106.

[32] Werner, K.H., Jazaieri, H., Goldin, P.R., Ziv, M., Heimberg, R.G., \& Gross, J.J. (2012). Self-compassion and social anxiety disorder. Anxiety Stress Coping, 25(5), 543-58.

[33] World Health Organisation (2019). Harmful use of alcohol, alcohol dependence and mental health conditions: a review of the evidence for their association and integrated treatment approaches.

[34] World Health Organisation (2018). Global status report on alcohol and health 2018. Geneva, WHO.

[35] World Health Organisation (2016). Health topics: Pregnancy. Geneva, Switzerland: WHO.

[36] World Health Organisation (2013). World Health Statistics 2013. Geneva, Switzerland: WHO.

[37] World Health Organization (2011). Global status report on alcohol and health: 2011. Geneva, Switzerland: Author

[38] World Health Organisation (2006). Global status report on alcohol 2006. Geneva, Switzerland: World Health Organisation

[39] World Health Organization (2004). Global Status Report on Alcohol 2004. Geneva: World Health Organization. 